\title{
Correction to: Equivalence Testing of Complex Particle Size Distribution Profiles Based on Earth Mover's Distance
}

\author{
Meng Hu, ${ }^{1}$ Xiaohui Jiang, ${ }^{1}$ Mohammad Absar, ${ }^{1}$ Stephanie Choi, ${ }^{1}$ Darby Kozak, ${ }^{1}$ Meiyu Shen, ${ }^{2}$ Yu-Ting Weng, ${ }^{2}$ \\ Liang Zhao, ${ }^{1,3}$ and Robert Lionberger ${ }^{1}$
}

Published online 14 August 2020

\section{Correction to: The AAPS Journal (2018) 20:62 https://doi.org/10.1208/s12248-018-0212-y}

On page 5, in the second paragraph, the authors inadvertently included inaccurate information for the description of the analytical method. The correct description of the method should read:

In this study, particle size was measured at $25{ }^{\circ} \mathrm{C}$ by dynamic light scattering (DLS) using the Zetasizer Nano ZSP instrument with measurement angle $173{ }^{\circ} \mathrm{C}$ (Malvern Instruments Inc., Westborough, MA, USA). All samples were diluted $\times 10$ using deionized water and placed in a quartz flow cell (ZEN0023) for size measurement using an autosampler. Ten (10) samples with six (6) measurements (or replicates) per sample were measured. The first three replicates were used for PSD comparison analysis. The attenuation index was set to 4 , and the measurement position was set to $4.2 \mathrm{~mm}$.

Reason for this change: Two series of experiments were conducted to measure the particle size distribution of the
RLD products by dynamic light scattering (DLS), with differences in number of lots and samples as well as in measurement settings. The results reported in the paper were obtained based on the data from the second series of experiment (with more lots and samples). However, we provided the description of the analytical method in the paper based on procedures from the first series of experiment. The revision in the manuscript above would correct this error.

On page 5, at the end of the first paragraph, please add:

The datasets analyzed in the current study are available in the figshare, https://doi.org/10.6084/ m9.figshare.12606080.v1.

Publisher's Note Springer Nature remains neutral with regard to jurisdictional claims in published maps and institutional affiliations.

The online version of the original article can be found at https:// doi.org/10.1208/s12248-018-0212-y

\footnotetext{
${ }^{1}$ Office of Research and Standards, Office of Generic Drugs, Center for Drug Evaluation and Research, Food and Drug Administration, Silver Spring, MD, USA.

${ }^{2}$ Office of Biostatistics, Office of Translational Sciences, Center for Drug Evaluation and Research, Food and Drug Administration, Silver Spring, MD, USA.

${ }^{3}$ To whom correspondence should be addressed. (e-mail: liang.zhao@fda.hhs.gov)
} 